\title{
GDNF secreted by pre-osteoclasts induces migration of bone marrow mesenchymal stem cells and stimulates osteogenesis
}

\author{
Sol $\mathrm{Yi}^{1,2}$, Jihee $\mathrm{Kim}^{1,2} \mathcal{E}$ Soo Young Lee ${ }^{1,2, *}$ \\ ${ }^{1}$ Department of Life Science, Ewha Womans University, Seoul 03760, ${ }^{2}$ The Research Center for Cellular Homeostasis, Ewha Womans \\ University, Seoul 03760, Korea
}

Bone resorption is linked to bone formation via temporal and spatial coupling within the remodeling cycle. Several lines of evidence point to the critical role of coupling factors derived from pre-osteoclasts (POCs) during the regulation of bone marrowderived mesenchymal stem cells (BMMSCs). However, the role of glial cell-derived neurotrophic factor (GDNF) in BMMSCs is not completely understood. Herein, we demonstrate the role of POC-derived GDNF in regulating the migration and osteogenic differentiation of BMMSCs. RNA sequencing revealed GDNF upregulation in POCs compared with monocytes/macrophages. Specifically, BMMSC migration was inhibited by a neutralizing antibody against GDNF in pre-osteoclast-conditioned medium (POC-CM), whereas treatment with a recombinant GDNF enhanced migration and osteogenic differentiation. In addition, POC-CM derived from GDNF knock-downed bone marrow macrophages suppressed BMMSC migration and osteogenic differentiation. SPP86, a small molecule inhibitor, inhibits BMMSC migration and osteogenic differentiation by targeting the receptor tyrosine kinase RET, which is recruited by GDNF into the GFR $\alpha 1$ complex. Overall, this study highlights the role of POC-derived GDNF in BMMSC migration and osteogenic differentiation, suggesting that GDNF regulates bone metabolism. [BMB Reports 2020; 53(12): 646-651]

\section{INTRODUCTION}

Continuous remodeling occurs in the skeletal system of adults to maintain bone homeostasis $(1,2)$. Cells associated with remodeling typically include osteoclasts and osteoblasts, both of which proliferate, migrate, and differentiate inside the bone marrow facilitated by various factors. Accordingly, bone remodel-

*Corresponding author. Tel: +82-2-3277-4257; Fax: +82-2-3277-3760; E-mail: leesy@ewha.ac.kr

https://doi.org/10.5483/BMBRep.2020.53.12.199

Received 17 September 2020, Revised 6 October 2020, Accepted 28 October 2020

Keywords: Bone homeostasis, Bone marrow mesenchymal stem cell, Cell migration, Glial cell-derived neurotrophic factor, Osteogenesis ing is known as "the coupling of osteoclasts and osteoblasts" because their action mechanisms communicate with each other (3-5). Bone resorption by osteoclasts is coupled to osteoblastinduced bone formation by endocrine and paracrine factors, via migration and differentiation of osteoblast precursors (6). The coupling factors derived from osteoclasts can be categorized into three groups. The first group, which involves transforming growth factor- $\beta$ (TGF- $\beta$ ) and insulin-like growth factor-I, is derived from the bone matrix $(6,7)$. The second group comprising factors, such as Eph receptor, is expressed on the cell membrane. The elements of the third group, which include platelet-derived growth factor-BB and sphingosine-1-phosphate, are secreted by osteoclasts and act on osteoblasts in a paracrine manner (5-7). Stimulation with macrophage colony-stimulating factor (M-CSF) and receptor activator of nuclear factor- $\kappa B$ ligand (RANKL) induces monocytes/macrophages into tartrateresistant acid phosphatase positive $\left(\mathrm{TRAP}^{+}\right)$mononuclear cells, which are then known as pre-osteoclasts (POCs) (8). Plateletderived growth factor-BB secreted by POCs affects bone marrowderived mesenchymal stem cells (BMMSCs), which are a major source of osteoblasts (9). BMMSCs are known to regulate bone marrow microenvironment, because their lineage affects the hematopoietic stem cell lineage, inducing their differentiation into osteoclasts (10).

Glial cell-derived neurotrophic factor (GDNF) is a member of the TGF- $\beta$ ligand superfamily, which includes GDNF, artemin, neurturin, and persephin (11). All ligands belonging to GDNF family bind differently to various complexes via GFR $\alpha 1-4$, a glycosylphosphoinositol linked subunit (12-14). GDNF exhibits high affinity for GFR $\alpha 1$ but typically uses the receptor tyrosine kinase RET as a signaling co-receptor (14). The small molecule inhibitor SPP86 is a selective inhibitor of RET tyrosine kinase, which induces PI3K/Akt and MAPK signaling that affects cell migration and differentiation (15-17). GDNF plays a major role as a growth factor that stimulates survival and proliferation of dopaminergic neurons, which are atrophied in Parkinson's disease (18).

In the present study, we investigated the functional role of POC-derived GDNF in BMMSC migration and osteogenic differentiation. The results suggest that GDNF acts as a bone-coupling factor linking bone resorption to bone formation.

ISSN: 1976-670X (electronic edition)

Copyright (c) 2020 by the The Korean Society for Biochemistry and Molecular Biology

(c) This is an open-access article distributed under the terms of the Creative Commons Attribution Non-Commercial License (http://creativecommons.org/licenses/by-nc/4.0) which permits unrestricted non-commercial use, distribution, and reproduction in any medium, provided the original work is properly cited. 


\section{RESULTS}

\section{GDNF is secreted from POC stimulated by RANKL}

To elucidate the potential mechanism by which POCs regulate BMMSCs, we cultured bone marrow monocytes/macrophages (BMMs) to induce their differentiation into POCs and obtained the conditioned medium derived from BMMs (control) and POCs (Supplementary Fig. 1A). BMMSC migration and osteogenic differentiation were induced significantly more in conditioned medium derived from POCs than in control conditioned medium from BMMs (Supplementary Fig. 1B, C), indicating that factors promoting BMMSC migration and osteogenesis were secreted in pre-osteoclast-conditioned medium (POC-CM).

We performed RNA sequencing of BMMs and POCs to identify factors from POC-CM regulating BMMSC migration and osteogenesis. We identified altered genes within the two groups: 14 secretion genes, 10 of which were merged up and 4 merged down (Fig. 1A). The genes that are known as osteoclast markers (19), including Ocstamp, Atp6v0d2, Acp5, Nfatc1, and Csf-1, were grouped together to establish osteoclast differentiation of BMMs (Fig. 1B). One of the increased factors, GDNF, is a member of the TGF- $\beta$ superfamily, and TGF- $\beta 1$
A

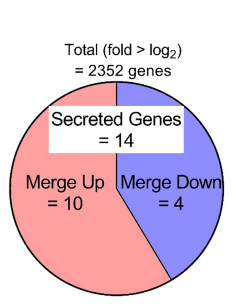

C

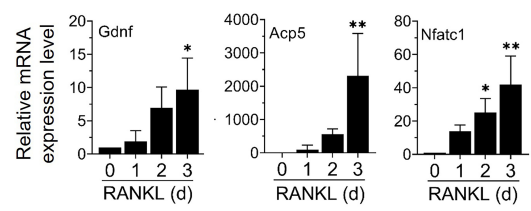

B

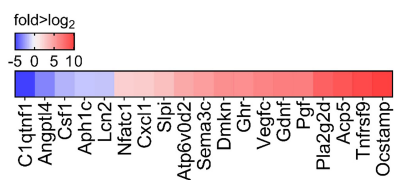

D
Fig. 1. GDNF is increased during osteoclast differentiation induced by RANKL. (A) A number of genes are differentially expressed in RANKL-stimulated BMMs on days 0 and 3, respectively. Based on the RNA sequencing results, 2,352 mRNAs were differentially expressed between BMMs and POCs. They included 14 mRNAs, including 10 upregulated and 4 downregulated mRNAs. (B) Heatmap of significantly up-regulated (red) or down-regulated (blue) genes in Venn diagram and osteoclast marker genes. (C) Gdnf, Acp5, and Nfatc1 mRNA levels of BMMs with RANKL stimulation for indicated days. Data were analyzed with qPCR. (D) The GDNF levels in the conditioned medium from RANKL-stimulated BMMs on days 0 and 3, respectively, were measured by ELISA. Mo/Mac CM, monocyte/macrophage-conditioned medium. POC CM, pre-osteoclastconditioned medium. Values are presented as the mean $+\mathrm{SD}$ (standard deviation). Student's t-test was used for statistical analysis $(\mathrm{C}$ and D). $* \mathrm{P}<0.05, * * \mathrm{P}<0.01$ reportedly regulates the reconstitution in bone microenvironment by inducing migration of BMMSCs $(11,20)$. We assume that POC-derived GDNF affects BMMSCs. The transcription of GDNF expression is increased during osteoclast formation by RANKL stimulation (Fig. 1C), and high levels of GDNF released to POC-CM were detected by enzyme-linked immunosorbent assay (ELISA) (Fig. 1D).

\section{GDNF induces migration and differentiation of BMMSCs}

We tested a neutralizing antibody against GDNF in the conditioned medium, and found that the neutralizing antibody abolished POC-CM-induced BMMSC migration (Fig. 2A, B).
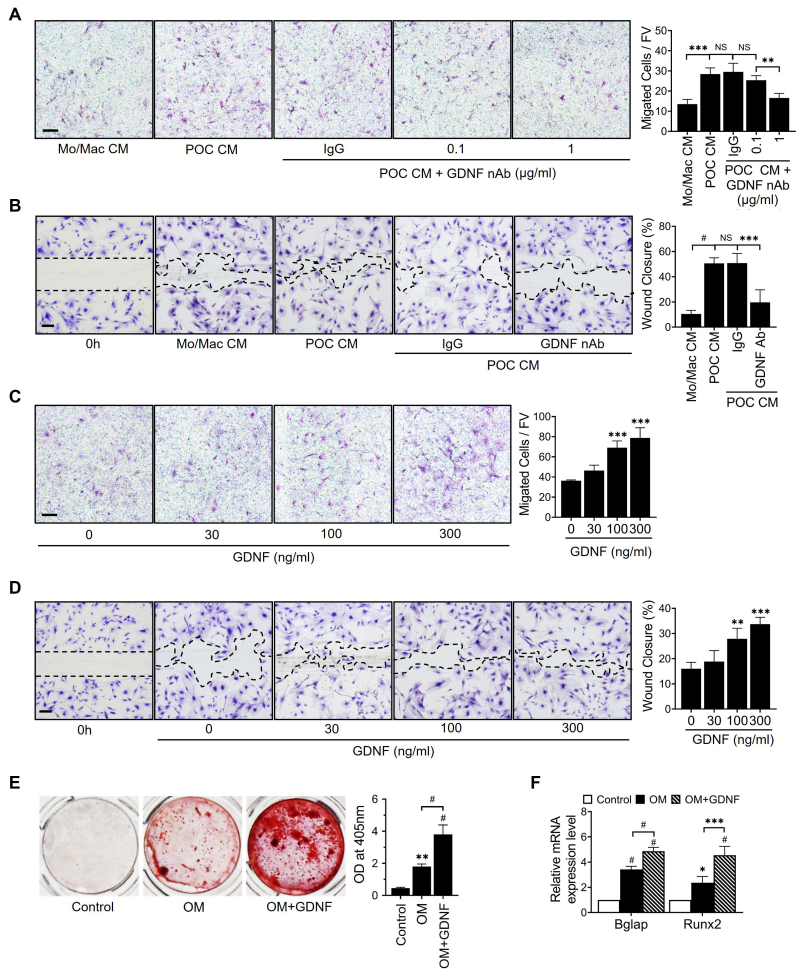

Fig. 2. GDNF-induced mobility and osteogenesis of BMMSCs. (A-D) Cell migration was evaluated by transwell migration and wound healing assays. BMMSCs were treated with (A, B) GDNF-neutralizing antibody (GDNF nAb) at indicated doses or $1 \mu \mathrm{g} / \mathrm{ml}$. Scale bars $=100 \mu \mathrm{m}$ and $200 \mu \mathrm{m}$. (C, D) Recombinant GDNF at indicated doses or $100 \mathrm{ng} / \mathrm{ml}$. Scale bars $=100 \mu \mathrm{m}$ and $200 \mu \mathrm{m}$. (E, F) Osteogenic differentiation was validated in BMMSCs in osteogenic medium (OM) supplemented with or without $100 \mathrm{ng} / \mathrm{ml}$ recombinant GDNF. Cells were stained with Alizarin red $\mathrm{S}$ solution, the concentration of which was measured as described in Materials and Methods (E). The expression of Bglap and Runx2 in osteoblasts was analyzed by qPCR (F). Values are presented as the mean \pm SD. A one-way ANOVA followed by Dunnett's multiple comparison test was used for statistical analysis (A and B). Values are mean \pm SD. Student's t-test was used for statistical analysis $(C, D, E$ and F). ${ }^{*} \mathrm{P}<0.05,{ }^{*} * \mathrm{P}<0.01,{ }^{*} * \mathrm{P}<0.005,{ }^{\#} \mathrm{P}<0.0001$. NS, Not significant. 
These results suggest that GDNF, whose function is not yet known in bone marrow environment, has an effect on BMMSCs. We hypothesized that GDNF directly affects BMMSC migration. Accordingly, recombinant GDNF treatment induces an increase in the migration of BMMSCs (Fig. 2C, D).

Next, we examined whether GDNF has an osteogenic potential. We treated BMMSCs with osteogenic media (OM) with or without GDNF. Alizarin red staining after $14 \mathrm{~d}$ increased BMMSC differentiation into osteoblasts (Fig. 2E). The transcription of bone formation marker genes showed that GDNF significantly stimulated Bglap and Runx2 expression $14 \mathrm{~d}$ after the differentiation of BMMSCs (Fig. 2F). Taken together, these results indicate that GDNF plays a distinct role in migration and osteogenic differentiation of BMMSCs.

\section{GDNF knockdown inhibits migration and osteogenic differentiation of BMMSCs}

We suppressed GDNF expression in POCs using small interfering RNA (siRNA) (Supplementary Fig. 2) and determined GDNF amounts by ELISA (Fig. 3A). We found that GDNF silencing in POCs reduced the secretion of GDNF into POC-CM. In addition, we observed reduced migration of BMMSCs using a transwell system and wound healing assays (Fig. 3B, C). GDNF knockdown in POCs markedly inhibited POC-CM-induced osteogenesis of BMMSCs (Fig. 3D). These results suggested that GDNF knockdown reduces the migration and osteogenic differentiation of BMMSCs by inhibiting GDNF secretion from POCs.

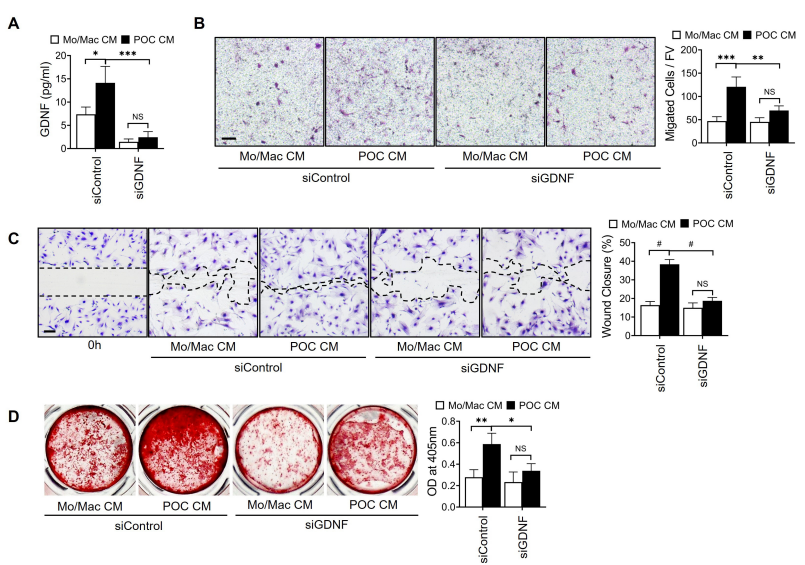

Fig. 3. GDNF knockdown in BMMs decreased the migration and differentiation of BMMSCs. (A) GDNF levels in conditioned medium derived from siGDNF-transfected BMMs were measured by ELISA. $(B, C)$ Cell migration was evaluated by transwell and wound healing assays with the conditioned medium. Scale bars $=100 \mu \mathrm{m}$ and $200 \mu \mathrm{m}$. (D) Osteogenesis was validated in BMMSCs in osteogenic medium with the indicated conditioned medium for $14 \mathrm{~d}$. Values are presented as the mean \pm SD. P values from two-way ANOVA were calculated followed by Tukey's multiple comparison test (A, $\mathrm{B}, \mathrm{C}$ and D). ${ }^{*} \mathrm{P}<0.05,{ }^{*} * \mathrm{P}<0.01,{ }^{* * * P}<0.005,{ }^{*} \mathrm{P}<0.0001$. NS, Not significant.

\section{GDNF-GFR $\alpha 1-R E T$ signaling mediates the migration and osteogenic differentiation of BMMSCs}

To investigate the mechanism of BMMSC migration stimulated by GDNF, we elucidated the signaling pathway of GDNF. As summarized in a schematic Supplementary Fig. 3A, SPP86 is a well-known small molecule inhibitor of the GDNF/GFR $\alpha 1 /$ RET pathway (15). We first established that BMMSCs carry receptors that potentially bind to GDNF and generate downstream signals. We demonstrated the expression of GDNF receptors, such as GFR $\alpha 1$ and RET, but not GFR $\alpha 2$ in BMMSCs (Supplementary Fig. 3B). The results suggested that GDNF binds to GFR $\alpha 1$ and recruits the co-receptor RET in BMMSCs.

Next, we analyzed the signal transduction pathway(s) that mediate the GDNF-induced migration of BMMSCs. Western blot analysis showed that POC-CM induced RET (Y1062) phosphorylation of BMMSCs within 15 min compared to control.
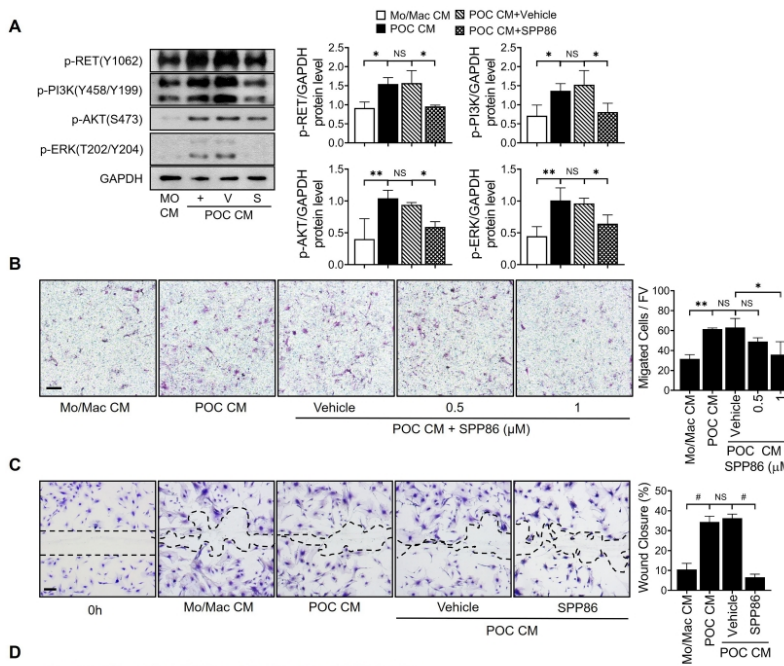

D

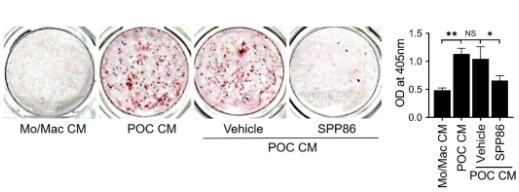

Fig. 4. Inhibition of GDNF signal suppressed migration and differentiation of BMMSCs. (A) Migration signals in BMMSCs inhibited by treatment with $1 \mu \mathrm{M}$ SPP86 for $3 \mathrm{~h}$ before stimulation and activated by conditioned medium for $15 \mathrm{~min}$ are shown using Western blot. The images were scanned with an office scanner and quantified by ImageJ. V, vehicle; S, SPP86. (B) Transwell migration assay was conducted to analyze the migration of BMMSCs in conditioned medium with or without the indicated doses of SPP86. Scale bar $=100 \mu \mathrm{m}$. (C) Wound healing assay was performed with conditioned medium and $1 \mu \mathrm{M}$ SPP86, and wound closure area was calculated by Image Pro. Scale bar $=200 \mu \mathrm{m}$. (D) Osteogenesis was validated in BMMSCs in conditioned medium supplemented with osteogenic factors, with or without $1 \mu \mathrm{M}$ SPP86. Values are presented as the mean \pm SD. $P$ values from one-way ANOVA were calculated followed by Dunnett's multiple comparison test (A, B, $\mathrm{C}$ and D). ${ }^{* P}<0.05,{ }^{*} \mathrm{P}<0.01,{ }^{\#} \mathrm{P}<0.0001$. NS, Not significant. 
Further, downstream signaling pathways, including phosphatidylinositol 3-kinase (PI3K), Akt and ERK are activated by POC-CM. Notably, the GDNF-RET signal inhibitor SPP86 abrogated the phosphorylation of RET and its downstream signaling pathway (Fig. 4A). Inhibition of GDNF/GFR $\alpha 1 /$ RET signaling pathway by SPP86 blocked POC CM-induced migration of BMMSCs (Fig. 4B, C) as well as osteogenic differentiation of BMMSCs (Fig. 4D). These results indicate that GFR $\alpha 1$-dependent activation of RET signaling mediates GDNF-induced BMMSC migration and osteogenic differentiation.

\section{DISCUSSION}

Osteoblast-lineage cells regulate osteoclast differentiation by secreting essential factors, including M-CSF, and RANKL (21). Osteoclasts also secrete factors known as clastokines that induce osteoblastic anabolic activity (22). The bidirectional osteoblastosteoclast communication couples the activities of bone-forming osteoblasts and bone-resorbing osteoclasts during bone turnover to maintain bone homeostasis $(23,24)$. BMMSC migration to the bone surface is an early step in bone formation $(10,25)$. In this regard, this study suggests that POC secretes GDNF, thereby playing an important role in regulating osteoblast formation not only by stimulating BMMSC migration, but also by inducing BMMSCs to promote osteogenesis.

GDNF is a small protein that potently promotes the survival of many types of neurons (26). Most notably, it facilitates the survival of dopaminergic and motor neurons (27). However, GDNF has other roles beyond the nervous system. GDNF controls the cell fate of undifferentiated spermatogonia in the testis (28). Furthermore, GDNF acts as a mesenchyme-derived signal that promotes ureteric branching in embryonic kidney (29). Our study validates and enhances the expression and function of GDNF synthesized and secreted by RANKL-stimulated osteoclast precursors. Extensive studies are needed to characterize its physiological significance in bone remodeling and to elucidate further the molecular mechanism of bone homeostasis regulated by POC-derived GDNF. In addition, the possible autocrine effect of GDNF secreted by POCs on osteoclast precursors require investigation. Toward this end, we analyzed the effect of recombinant GDNF on osteoclast differentiation. Despite the increasing GDNF concentrations, no change in the number of TRAP $^{+}$multinucleated cells, known as osteoclasts was detected, indicating that GDNF does not directly affect RANKL-induced osteoclast differentiation (Supplementary Fig. 4).

Taken together, our studies suggest that GDNF mediates osteoclast-osteoblast crosstalk by regulating BMMSC migration and osteogenesis. These findings indicate a new role of POCproduced GDNF as a potential clastokine. While further studies are necessary to fully elucidate the mechanism of action of GDNF in bone remodeling and to determine the specific role of POC-derived GDNF in vivo, it is clear that GDNF acts directly on BMMSCs to potentially modulate bone homeostasis.

\section{MATERIALS AND METHODS}

\section{Primary cell culture}

We used tibia and femur of 4- to 5-week-old male C57BL/6J mice obtained from the Animal Care Committee of Ewha Laboratory Animal Genomics Center to isolate primary cells. Bone marrow-derived macrophages (BMMs) and bone marrow mesenchymal stem cells (BMMSCs) were prepared as previously described $(30,31)$. The cells were cultured in $\alpha$-MEM (Hyclone, USA) supplemented with $10 \%$ FBS (Gibco, USA) and $1 \%$ penicillin and streptomycin (Gibco, USA).

\section{Conditioned medium}

We prepared POC-CM from osteoclasts. Upon incubation of BMM with $30 \mathrm{ng} / \mathrm{ml} \mathrm{M-CSF}$ and $150 \mathrm{ng} / \mathrm{ml}$ RANKL in 6-well plates $\left(3 \times 10^{5}\right.$ cells per well), all cells turned into POCs after $2 \mathrm{~d}$ or $3 \mathrm{~d}$ of culture. We harvested the conditioned medium on days 2 and 3 , followed by centrifugation at $700 \mathrm{~g}$ for 10 $\min$ at $4^{\circ} \mathrm{C}$ to eliminate floating cells. We then aliquoted the conditioned medium and stored it at $-20^{\circ} \mathrm{C}$ until use. In some experiments, we added $1 \mu \mathrm{g} / \mathrm{ml}$ of goat-lgG (Santa Cruz Biotechnology, USA) or GDNF-neutralizing antibody (R\&D, USA) and a GDNF-RET inhibitor, $1 \mu \mathrm{M}$ SPP86 (TOCRIS, USA) to the conditioned medium. The day after BMM plating, we harvested the medium with negative control, monocyte and macrophageconditioned medium (Mo/Mac-CM).

\section{Real-time quantitative polymerase chain reaction}

Total RNA was extracted using TRIzol (iNtRON Biotechnology, Korea) according to the manufacturer's instructions. Total RNA was reverse transcribed into cDNA using a Diastar Reverse Transcriptional Kit (BIOFACT, Korea). Polymerase chain reaction (PCR) amplification was conducted using a SYBR Hi-ROX Kit (Bioline, UK). The reaction was performed $95^{\circ} \mathrm{C}$ for $3 \mathrm{~min}$, then 40 cycles of $95^{\circ} \mathrm{C}$ for $15 \mathrm{sec}, 60^{\circ} \mathrm{C}$ for $30 \mathrm{sec}$, and $70^{\circ} \mathrm{C}$ for 60 sec. The ABI PRISM 7300 system (Applied Biosystems, USA) was used to amplify DNA and detect the PCR products. Each experiment was conducted in triplicate, and the expression levels of the target genes were normalized to those of actin. The melting curve was analyzed to ensure that only the desired PCR product was present. The primer sequences used for genes were: Gdnf (Forward 5'-TCCTGACCAGTTTGATGACG3', Reverse 5'-CCGATTCCTCTCTCTTCGAG-3'), Acp5 (Forward 5'-CATTGTTAGCCACATACGG-3', Reverse 5'-ACTCAGCACAT AGCCCACAC-3'), Nfatc1 (Forward 5'-CCAGAAAATAACATGCG AGCC-3', Reverse 5'-GTGGGATGTGAACTCGGAAG-3'), Bglap (Forward 5'-CTGACCTCACAGATCCCAAGC-3', Reverse 5'-TG GTCTGATAGCTCGTCACAAG-3'), Runx2 (Forward 5'-GACTG TGGTTACCGTCATGGC-3', Reverse 5'-ACTTGGTTTTTCATAACAG CGGA-3'), Gfra1 (Forward 5'-AGAAGCAGTTTCACCCAG-3', Reverse 5'-ATCATCACCACCACCATC-3'), Gfra2 (Forward 5'-TGCC TCTTCTTCTITITAGGGACA-3', Reverse 5'-GCAGTTGTCGTTCA GGTTGC-3'), Ret (Forward 5'-ACACGGCTGCATGAGAATGAC3', Reverse 5'-TGCTGAGCTGTTCCCAGGAA-3'), and $\beta$-actin (For- 
ward 5'-GTGACGTTGACATCCGTAAAGA-3', Reverse 5'-GCC GGACTCATCGTACTCC-3').

\section{Transwell migration assay of BMMSCs}

We assessed cell migration in transwell 24-well plates (Corning, USA) with $8 \mu \mathrm{m}$ pore filters. We seeded $3 \times 10^{4}$ cells per well in the upper chambers with serum-free medium followed by incubation with $\alpha$-MEM (Hyclone, USA) including 10\% FBS (Gibco, USA) and $1 \%$ penicillin and streptomycin (Gibco, USA) or conditioned medium in the lower chambers. After $5 \mathrm{~h}$ incubation, we fixed the cells with $4 \%$ formaldehyde (Sigma-Aldrich, USA) for $30 \mathrm{~min}$ and then deleted the cells on the upper surface of each filter with cotton swabs. We stained the cells that migrated through the pores to the lower surface with $0.2 \%$ crystal violet (Sigma-Aldrich, USA) for another $30 \mathrm{~min}$. We acquired images with a light microscope at $\times 100$ magnification and quantified them by counting five random fields per well using a microscope (Olympus, Japan) at $\times 200$ magnification.

\section{Wound healing assay}

Cells were seeded on 6 -well plates at $5 \times 10^{5}$ cells per well. After $24 \mathrm{~h}$, the cells were washed with PBS, and wounds were created using a sterile $200 \mu \mathrm{l}$ pipette tip. The cells were then washed with PBS and incubated in a medium described in the figure legends. After the incubation, the cells were fixed with $4 \%$ formaldehyde for $30 \mathrm{~min}$ and stained with $0.2 \%$ crystal violet (Sigma-Aldrich, USA) for another $30 \mathrm{~min}$. Three visual fields were randomly selected to observe the migration at the wound site. The images were obtained with a light microscope at $\times 40$ magnification, and the wound closure area was calculated as previously described (32).

\section{ELISA}

We performed GDNF enzyme-linked immunosorbent assay (ELISA) of the conditioned medium from BMMs and POCs after days 0 and 3, respectively, following RANKL treatment using a mouse GDNF ELISA kit (Abcam, USA). We performed all ELISAs according to the manufacturer's instructions. The conditioned medium from bone marrow macrophages or the osteoclast culture was harvested and concentrated using Amicon Ultra centrifugal filter for $10 \mathrm{kDa}$ (Millipore, USA) as previously reported (33).

\section{Osteogenic differentiation}

We seeded BMMSCs in a 48-well culture plate at a density of $2 \times 10^{4}$ cells per well. To induce osteogenic differentiation, the osteogenic medium was supplemented with $50 \mu \mathrm{g} / \mathrm{ml}$ ascorbic acid, $5 \mathrm{mM} \beta$-glycerophosphate, and $10 \mathrm{nM}$ dexamethasone (all from Sigma-Aldrich) used with conditioned medium or in recombinant GDNF treatment. The culture medium was replaced every 2 to 3 days. All cells were cultured in the cell incubator $\left(37^{\circ} \mathrm{C}, 5 \% \mathrm{CO}_{2}\right)$. After $14 \mathrm{~d}$, we evaluated the cell matrix mineralization by alizarin red $\mathrm{S}$ staining with $2 \%$ of Alizarin red $\mathrm{S}$ (Sigma-Aldrich) dissolved in PBS and its $\mathrm{pH}$ was adjusted to 4.2. We measured the absorbance of the dissolved extracts to quantify the insoluble alizarin in $10 \%$ acetic acid. All measurements were conducted at an absorbance of $405 \mathrm{~nm}$ by SpectraMax 190 (Molecular Devices, USA).

\section{Small interfering RNA (siRNA) transfection}

To silence the expression of GDNF, we treated BMMs with 50 $\mu \mathrm{M}$ siRNA or negative control. The siRNA sequence was used to incubate transfected BMMs for $4 \mathrm{~h}$ and culture them for 2 to $3 \mathrm{~d}$ with new medium supplemented with $30 \mathrm{ng} / \mathrm{ml}$ and 150 $\mathrm{ng} / \mathrm{ml}$ RANKL. The target sequences were: siControl: forward 5'-CCUCGUGCCGUUCCAUCAGGUAGUU-3' and reverse 5'CUACCUGAUGGAACGGCACGAGGUU-3'. siGDNF forward 5'-CCAAUAUGCCUGAAGAUUAUCCUGAUU-3' and reverse

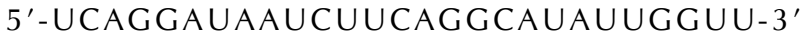
(Genolution, USA).

\section{Western blot analysis}

The BMMSCs were plated in $30 \mathrm{~mm}$ culture plates at $5 \times 10^{5}$ cells per plate. After $24 \mathrm{~h}$, the incubated medium was changed with $\alpha$-MEM (Hyclone, USA) supplemented with $0.1 \%$ FBS (Gibco, USA) and $1 \%$ penicillin and streptomycin (Gibco, USA) for starvation $3 \mathrm{~h}$ before signal stimulation. The cells were lysed in a buffer containing $50 \mathrm{mM}$ Tris-Cl (pH 7.4), $150 \mathrm{mM}$ $\mathrm{NaCl}, 1 \mathrm{mM}$ EDTA, $0.2 \%$ sodium deoxycholic acid, $0.5 \%$ NP40, proteinase inhibitors ( $1 \mathrm{mM}$ PMSF and $1 \mu \mathrm{g} / \mathrm{ml}$ leupeptin and aprotinin), and phosphatase inhibitors ( $1 \mathrm{mM} \mathrm{NaVO} 4$ and $1 \mathrm{mM} \mathrm{NaF})$. Cell lysates or immunoprecipitated proteins were separated using $8 \%$ SDS-polyacrylamide gels as previously reported (34). The membranes were immunoblotted with primary antibodies against phospho-RET, phospho-PI3K, phospho-AKT, phospho-ERK (Cell Signaling Technology, Inc., USA), GAPDH (Santa Cruz Biotechnology, USA), and secondary antibodies. Proteins were detected using an ECL detection Kit (Bio-Rad Laboratories, USA). Representative western blots and quantification (shown in the bar graph) of the indicated protein/control ratio in the cell lysates using ImageJ are shown in Fig. 4A.

\section{Statistical analysis \\ Data are expressed as the mean \pm SD of at least three indepen- dent experiments. Statistical analyses were performed using GraphPad Prism 8 (GraphPad Software Inc., USA). Group differ- ences were analyzed by Student's t-test. One-way ANOVA followed by Dunnett's multiple comparison test and two-way ANOVA followed by Tukey's multiple comparison test were used to analyze differences among the groups. ${ }^{*} \mathrm{P}<0.05$, **P $<$ $0.01, * * * \mathrm{P}<0.005,{ }^{\#} \mathrm{P}<0.001$ were considered statistically significant.}

\section{ACKNOWLEDGEMENTS}

This work was supported by grants from the National Research Foundation of Korea (2019R1A5A6099645; 2019R1A6C1010020) and by the Ewha Womans University Research Grant (1-20171157-001). 


\section{CONFLICTS OF INTEREST}

The authors have no conflicting interests.

\section{REFERENCES}

1. Frost HM (1969). Tetracycline-based histological analysis of bone remodeling. Calcif Tissue Res 3, 211-237

2. Eriksen EF (2010) Cellular mechanisms of bone remodeling. Rev Endocr Metab Disord 11, 219-227

3. Parfitt AM (1982). The coupling of bone formation to bone resorption: a critical analysis of the concept and of its relevance to the pathogenesis of osteoporosis. Metab Bone Dis Relat Res 4, 1-6

4. Martin TJ and Sims NA (2005) Osteoclast-derived activity in the coupling of bone formation to resorption. Trends Mol Med 11, 76-81

5. Ikeda K and Takeshita S (2014) Factors and mechanisms involved in the coupling from bone resorption to formation: how osteoclasts talk to osteoblasts. J Bone Metab 21, 163-167

6. Han Y, You X, Xing W, Zhang Z and Zou W (2018) Paracrine and endocrine actions of bone - the functions of secretory proteins from osteoblasts, osteocytes, and osteoclasts. Bone Res 6, 1-11

7. Tang Y, Wu X, Lei W et al (2009) TGF- $\beta 1$-induced migration of bone mesenchymal stem cells couples bone resorption with formation. Nat Med 15, 757-765

8. Woo J-T, Kato M, Takami M and Nagai K (2000) Receptor activator of $\mathrm{NF}-\kappa \mathrm{B}$ ligand induces the fusion of mononuclear preosteoclasts into multinucleated osteoclasts. Cytotechnology 33, 203-211

9. Xie H, Cui Z, Wang L et al (2014) PDGF-BB secreted by preosteoclasts induces angiogenesis during coupling with osteogenesis. Nat Med 20, 1270-1278

10. Su P, Tian Y, Yang C et al (2018) Mesenchymal stem cell migration during bone formation and bone diseases therapy. Int J Mol Sci 19, 2343

11. Saarma M (2000) GDNF - a stranger in the TGF- $\beta$ superfamily? Eur J Biochem 267, 6968-6971

12. Airaksinen MS, Titievsky A and Saarma M (1999) GDNF family neurotrophic factor signaling: four masters, one servant? Mol Cell Neurosci 13, 313-325

13. Airaksinen MS and Saarma M (2002) The GDNF family: signalling, biological functions and therapeutic value. Nature Rev Neurosci 3, 383-394

14. Amoresano A, Incoronato M, Monti G, Pucci P, De Franciscis $V$ and Cerchia L (2005) Direct interactions among Ret, GDNF and GFR 1 molecules reveal new insights into the assembly of a functional three-protein complex. Cell Signal $17,717-727$

15. Alao JP, Michlikova S, Dinér P, Grøtli $M$ and Sunnerhagen P (2014) Selective inhibition of RET mediated cell proliferation in vitro by the kinase inhibitor SPP86. BMC Cancer 14,853

16. Castellano E, Molina-Arcas M, Krygowska AA et al (2016) RAS signalling through PI3-Kinase controls cell migration via modulation of Reelin expression. Nat Commun 7, 1-13

17. Crupi MJ, Maritan SM, Reyes-Alvarez E et al (2019) GGA3mediated recycling of the RET receptor tyrosine kinase con- tributes to cell migration and invasion. Oncogene 39, 13611377

18. Rangasamy SB, Soderstrom K, Bakay RA and Kordower JH (2010) Neurotrophic factor therapy for Parkinson's disease; in Progress in brain research 237-264, Elsevier

19. Yang $M$, Birnbaum MJ, MacKay CA, Mason-Savas A, Thompson B and Odgren PR (2008) Osteoclast stimulatory transmembrane protein (OC-STAMP), a novel protein induced by RANKL that promotes osteoclast differentiation. J Cell Physiol 215, 497-505

20. Xian L, Wu X, Pang L et al (2012) Matrix IGF-1 maintains bone mass by activation of mTOR in mesenchymal stem cells. Nat Med 18, 1095

21. Yamamoto $Y$, Udagawa N, Matsuura S et al (2006) Osteoblasts provide a suitable microenvironment for the action of receptor activator of nuclear factor- $\mathrm{\kappa B}$ ligand. Endocrinology 147, 3366-3374

22. Martin TJ (2014) Bone biology and anabolic therapies for bone: current status and future prospects. J Bone Metab 21, 8-20

23. Kreja, L., Brenner, R. E., Tautzenberger et al (2010) Nonresorbing osteoclasts induce migration and osteogenic differentiation of mesenchymal stem cells. J Cell Biochem 109, 347-355.

24. Kim I, Kim JH, Kim K, Seong S and Kim N (2019) The IRF2BP2-KLF2 axis regulates osteoclast and osteoblast differentiation. BMB Rep 52, 469-474

25. Wang Y, Chen X, Yin Y and Li S (2018) Human amnionderived mesenchymal stem cells induced osteogenesis and angiogenesis in human adipose-derived stem cells via ERK1/2 MAPK signaling pathway. BMB Rep 51, 194-199

26. Lin LF, Doherty DH, Lile JD, Bektesh S and Collins F (1993) GDNF: a glial cell line-derived neurotrophic factor for midbrain dopaminergic neurons. Science 260, 1130-1132

27. Oppenheim RW, Houenou LJ, Johnson JE et al (1995) Developing motor neurons rescued from programmed and axotomy-induced cell death by GDNF. Nature 373, 344-346

28. Chen L-Y, Willis WD and Eddy EM (2016) Targeting the Gdnf gene in peritubular myoid cells disrupts undifferentiated spermatogonial cell development. Proc Natl Acad Sci U S A 113, 1829-1834

29. Pichel JG, Shen L, Sheng HZ et al (1996) Defects in enteric innervation and kidney development in mice lacking GDNF. Nature 382, 73-76

30. Jang HD, Shin JH, Park DR et al (2011) Inactivation of glycogen synthase kinase- $3 \beta$ is required for osteoclast differentiation. J Biol Chem 286, 39043-39050

31. Zhu H, Guo Z-K, Jiang X-X et al (2010) A protocol for isolation and culture of mesenchymal stem cells from mouse compact bone. Nat Protoc 5, 550

32. Yue PY, Leung EP, Mak NK, and Wong RN (2010) A simplified method for quantifying cell migration/wound healing in 96-well plates. J Biomol Screen 15, 427-433.

33. Xiao N, Yu WY and Liu D (2018) Glial cell-derived neurotrophic factor promotes dental pulp stem cell migration. J Tissue Eng Regen Med 12, 705-714

34. Park JH, Jeong E, Lin J et al (2019) RACK1 interaction with c-Src is essential for osteoclast function. Exp Mol Med 51, 1-9 